\title{
Über das Individuelle, Subjektive und Integrative in der Komplementärmedizin
}

\author{
Reinhard Saller \\ Institut für Naturheilkunde, Department für Innere Medizin, Universitätsspital Zürich, Schweiz
}

Attraktivität, Gebrauch und offensichtlicher Nutzen komplementärmedizinischer Vorgehensweisen erscheinen eigentümlich, wenn man nur die verfügbaren quantitativen Erhebungen und Fragebogendaten heranzieht, um die meist dezidierten Patientenentscheidungen für Komplementärmedizin zu erklären. Es wird vielfach vermutet, dass die Erwartung von individualisierten Behandlungsweisen und deren partielle Gleichsetzung mit Komplementärmedizin ein entscheidender Grund für die patienteneigene Einbeziehung komplementärmedizinischer Methoden und Ansätze sei. Individualisierung ist jedoch keineswegs nur ein Charakteristikum komplementärmedizinischer Behandlungen. Gerade auch die Erwartungen an die molekulare Medizin gründen sich auf eine Individualisierung der Behandlungswege, d.h. eine exakte Anpassung an die Individualität von Krankheit und Patient. Individualisierung bedeutet aber nicht notwendigerweise, dass Patienten als Subjekte eingebunden sind, d.h. als handelnde und (mit-) gestaltende Personen. Einer der wesentlichen Gesichtspunkte für das Bedürfnis von Patienten, flexibel und zeitlich variabel komplementärmedizinische Möglichkeiten in ihre häufig vielschichtigen Behandlungspakete einzubinden, ist eine dementsprechende Subjektivität, die Subjektivität des Kranken inmitten der Objektivität der Daten. Die Integration der Subjektivität ist ein wesentlicher Faktor jener Ganzheitlichkeit, die gewünscht wird. Ganzheitlichkeit bedeutet ja nicht, undifferenziert möglichst viel zu machen, sondern jeweils krankheitsbezogen und personal eine mehrdimensionale Behandlung mitwählen und gestalten zu können. Damit bekommt auch vieles von dem, was unter «Placebo» diskutiert wird, eine operationale und nicht diskriminierende Ausrichtung: eine gezielte und patientenspezifische Mobilisierung von Selbstheilungsmöglichkeiten von Individuum und Subjekt. Es erscheint dann allerdings auch notwendig, den bisherigen eingeschränkten Begriff von Evidenz zu überdenken. Er müsste eine neue Plastizität erhalten [1]. Man könnte über eine «Ar- tenvielfalt» von Evidenzen spekulieren, die nicht a priori starr hierarchisch geordnet sind. Die wiederentdeckte «Narration» in der Medizin weist ebenfalls in diese Richtung, desgleichen das mögliche Spannungsfeld zwischen naturwissenschaftlicher Exaktheit in der Medizin und der Medizin als einer Beschäftigung mit Bedeutungen.

Viele Patienten sind der Überzeugung, dass individuelle, subjektive und lebensgeschichtliche Aspekte durch komplementärmedizinische Krankheitsdeutungen und Therapieverfahren aufgegriffen werden. Die relativ freie Theorie- und Methodenwahl und die aktive Miteinbeziehung in das therapeutische Vorgehen qualifiziert die Patienten nicht nur als «Behandelte», sondern auch als «Handelnde» [2]. Subjektiv überzeugende Deutungs- und Handlungsangebote helfen dem Patienten $\mathrm{zu}$ therapeutisch relevanten Selbstinterpretationen und Reaktionsmöglichkeiten. So tragen medikamentöse Therapien nicht nur die Inhaltsstoffe, die das jeweilige Medikament enthält. Sie transportieren neben den molekularen Möglichkeiten Ideen, Symbole, Zeichen sowie Vorstellungen von Krankheit und Genesung. In der Phytotherapie z.B. begegnen sich zeitlich, regional, historisch, kulturell und geografisch gesehen verschiedene Therapierichtungen, Medizinkonzepte und Krankheitslehren und ein bunter Ideen- und Erfahrungsschatz mehrerer Jahrtausende mit vielfältigen objektiven und subjektiven Therapiemöglichkeiten. Unter anderem wären Assoziationen zu einer Art «Biologie der Subjekte» möglich. In diesem Zusammenhang müsste man auch reflektieren, inwieweit Patienten eine integrative Medizin erwarten, was auch immer verschiedene, zum Teil sehr ausdifferenzierte Konzepte darunter verstehen. In den letzten Jahren wird immer mehr Methoden und Mitteln die Bezeichnung und damit die erwartete Eigenschaft «integrativ» verliehen. Unter dem Gesichtspunkt des Patienten als Subjekt könnte es sinnvoller sein (und begrifflich wäre dies ohnedies präziser), nicht die einzelnen Methoden als integrativ zu klassifizieren oder auszuzeichnen,

\begin{tabular}{|c|c|}
\hline KARGER & (c) 2008 S. Karger GmbH, Freiburg \\
\hline $\begin{array}{l}\text { Fax +49 } 7614520714 \\
\text { E-mail Information@Karger.de } \\
\text { www.karger.com }\end{array}$ & $\begin{array}{l}\text { Accessible online at: } \\
\text { www.karger.com/fok }\end{array}$ \\
\hline
\end{tabular}

Prof. Dr. med. Reinhard Saller Institute für Naturheilkunde

Department für Innere Medizin, Universitätsspital Zürich

Rämistrasse 100, CH-8091 Zürich, Schweiz

Tel. +41 44 255-2460, Fax -4394

E-mail reinhard.saller@usz.ch 
sondern die patientenbezogenen Vorgehensweisen. Es ist unmittelbar einsichtig, dass z.B. Krankenhäuser einen integrativen Ansatz wählen, Krankenkassen ein integratives Vorgehen ermöglichen oder Medizinsysteme die entsprechenden Rahmenbedingungen garantieren. Mit einem solchen Verständnis behalten die einzelnen Disziplinen ihre Authentizität. Sie sind aber gehalten, patienten- und krankheitsbezogen flexibel im Rahmen einer garantierten, aber selbstverständlich wohlüberlegten medizinischen Vielfalt zusammenzuarbeiten. Bezogen auf eine gesichtete Komplementärmedizin würde dies bedeuten, dass entsprechende Angebote vorhanden bzw. erreichbar sein müssten.

\section{Literatur}

1 Coulter ID: Evidence based complementary and alternative medicine: Promises and problems. Forsch Komplementärmed 2007;14:102-108.

2 Saller R: Gesichtete Komplementärmedizin; in Streit E, Rist L (Hrsg): Ethik und Wissenschaft in der anthroposophischen Medizin. Beiträge zu einer Erneuerung der Medizin. Bern, Lang, 2006, pp 9-4. 